\title{
Radiomics Signatures of Computed Tomography Imaging for Predicting Resection Margin Status in Pancreatic Head Adenocarcinoma
}

Jinheng Liu

Sichuan University West China Hospital

Xubao Liu

Sichuan University West china hospital

Jiajun Qiu

Sichuan University West China Hospital

Yanting Wang

Sichuan University West China School of Public Health

Wei Huang

Sichuan University West China Hospital

Nengwen Ke ( $\nabla$ kenengwen@scu.edu.cn )

Sichuan University West China Hospital https://orcid.org/0000-0002-7135-2244

Research article

Keywords: pancreatic adenocarcinoma; resection margin; Computed Tomography; quantitative analysis

Posted Date: August 17th, 2020

DOI: https://doi.org/10.21203/rs.3.rs-46691/v1

License: () (1) This work is licensed under a Creative Commons Attribution 4.0 International License.

Read Full License 


\section{Abstract}

Background: To identify preoperative computed tomography radiomics texture features which correlate with resection margin status and prognosis in resected pancreatic head adenocarcinoma.

Methods: Improved prognostication methods utilizing novel non-invasive radiomic techniques may accurately predict resection margin status preoperatively. In an ongoing concerning pancreatic head adenocarcinoma, the venous enhanced CT images of 86 patients who underwent pancreaticoduodenectomy were selected, and the resection margin ( $>1 \mathrm{~mm}$ or $\leq 1 \mathrm{~mm}$ ) was identified by pathological examination. Three regions of interests (ROIs) were then taken from superior to inferior facing the superior mesenteric vein and artery. Subsequent Laplacian-Dirichlet based texture analysis methods extracting algorithm flows of texture features within ROls were analyzed and assessed in relation to patient prognosis.

Results: Patients with $>1 \mathrm{~mm}$ resection margin had an overall improved survival compared to $\leq 1 \mathrm{~mm}(P<$ 0.05). Distance 1 and 2 of Gray level co-occurrence matrix, high Gray-level run emphasis of run-length matrix and average filter of wavelet transform (all $P<0.05$ ) were correlated with resection margin status (Area under the curve was 0.784 , sensitivity was $75 \%$ and specificity was $79 \%$ ). The energy of wavelet transform, the measure of smoothness of histogram and the variance in 2 direction of Gabor transform are independent predictors of overall survival prognosis, independent of resection margin.

Conclusions: Resection margin status ( $>1 \mathrm{~mm}$ vs $\leq 1 \mathrm{~mm}$ ) is a key prognostic factor in pancreatic adenocarcinoma and CT radiomic analysis have the potential to predict resection margin status preoperatively, and the radiomic labels may improve selection neoadjucant therapy.

Trial registration: Clinicaltrials.gov/ct2/show/NCT02928081.

\section{Background}

The incidence of pancreatic cancer is estimated to be 8.14 with mortality close by at 6.92 deaths per 100,000 person-years. ${ }^{1}$ Age-standardized mortality for pancreatic cancer in China during 1990-2017 increased more than $20 \%^{2}$ and it is expected to rise to the second leading cause of cancer-related death by $2030 .{ }^{3}$ Despite a doubling of the 5 -year survival rate for patients with pancreatic cancer over the last two decades (4\% in 2001 vs $9 \%$ 2019), 3 the prognosis remains poor, with surgical resection the only potentially curative treatment. ${ }^{5}$ The aim of any "curative" resection for pancreatic cancer is to achieve free margins. ${ }^{6,7}$ Recently, several randomized controlled trials (RCTs) indicated that a resection margin of > $1 \mathrm{~mm}$ yields a better survival rate, compared with a resection margin of $\leq 1 \mathrm{~mm}, 8,9,10$ especially for the "real resection margin"- the portal vein-superior mesenteric vein margin and the superior mesenteric artery margin. 8,9 When the resection margin was $\leq 1 \mathrm{~mm}$, there was no difference in survival rate compared to patients with R1 resection (presence of tumor cells at the surface of the resection margins). ${ }^{8}$ Delpero et al. also observed that the patients who got $\leq 1 \mathrm{~mm}$ resection margin might benefit from 
neoadjucant therapy, especially for the patients with lymph node positive. ${ }^{8}$ These results implied that resection after neoadjuvant therapy would be better choice for patients who got $\leq 1 \mathrm{~mm}$ resection margin. However, there was no useful method to predict the margin preoperatively till now.

"Radiomics", which is defined as the high-throughput extraction of image features from radiographic images, has potential to provide a detailed pre-operative evaluation method of tumor heterogeneity. ${ }^{11-17}$ This method of imaging analysis utilizes algorithms to derive image texture. At present, the most commonly used image texture analysis methods are first and second order statistical method analyses of image pixels and their neighborhood gray level. ${ }^{15,18}$ The first order method is to evaluate the grayscale frequency distribution of the pixel intensity histogram in a specific region of interest (ROI), including average intensity, threshold, entropy, standard deviation, skewness, and kurtosis. ${ }^{19}$ The second order method is a texture feature extraction method that analyzes the combined probability distribution of specific pixel pairs based on the Gray-level co-occurrence matrix (second order entropy, energy, uniformity, difference and correlation). ${ }^{19}$ Through the analysis of tumor texture features in this manner, meaningful textural parameters have been correlated with disease diagnosis, pathological grading, and therapeutic effect prediction and survival rate. ${ }^{19} 2021$ Previous imaging studies of pancreatic diseases mainly focused on the analysis of the texture features of pancreatic tumors to distinguish pancreatic cancer from chronic pancreatitis or neuroendocrine tumors. ${ }^{22-26}$ However, up to now, no texture analysis of the resection margin for pancreatic head adenocarcinoma has been performed to identify the characteristics of resection margins $>1 \mathrm{~mm}$ or $\leq 1 \mathrm{~mm}$ and their relation to resection margin and postoperative prognosis.

In this study, we aimed to use a prospective clinical database to evaluate the probability that CT radiomic labels could identify the resection margins of the pancreatic head adenocarcinoma $>1 \mathrm{~mm}$ or $\leq 1 \mathrm{~mm}$ before surgery.

\section{Methods}

\section{Patients and study design}

The study protocol was approved by the Ethics Committee of West China Hospital of Sichuan University (2016:122). Patients diagnosed with pancreatic duct adenocarcinoma who received informed consent were prospectively enrolled into an ongoing registered RCT (This is an RCT about Standard Versus Extended Lymphadenectomy in Pancreatoduodenectomy for Patients with Pancreatic Head Adenocarcinoma.Clinicaltrials.gov/ct2/show/NCT02928081.) All patients were screened during October 2015 to March 2018. All patients underwent preoperative imaging examination, pancreaticoduodenectomy, and postoperative pathological examination (confirmative diagnosis). No patients got neoadjuvant chemotherapy, adjuvant therapy was strongly recommended to all patients after surgery. Their clinical data, CT images, surgical procedures, resection margins and pathological results were collected. Patients with alternative diagnoses, radiological imaging performed at another referring 
hospital or of poor quality were excluded. All resection margins were stained in accordance with pathological examination criteria (Fig. 1A). According to the portal vein-superior mesenteric vein margin and the superior mesenteric artery margin postoperative pathological examination results, patients were then divided into either the $>1 \mathrm{~mm}$ resection margin group or the $\leq 1 \mathrm{~mm}$ resection margin group (carcinoma was found within $1 \mathrm{~mm}$ of the resection margin).

\section{Abdominal CT Protocol}

An abdominal plain scan and enhanced scan were performed with a 64-slice spiral Siemens CT (Munich, Germany). Collimator $0.625 \mathrm{~mm}$, FOV $450 \mathrm{~mm}$ x $350 \mathrm{~mm}$, tube voltage 120-140 kV, tube current 210 $\mathrm{mAs}$, layer thickness $2.0 \mathrm{~mm}$. Contrast agent iodohydrin was injected into the basilic vein for enhanced scanning, with a flow rate of $2.0-3.0 \mathrm{ml} / \mathrm{s}$ and a dose of $60-100 \mathrm{~mL}$, and a delay of $70 \mathrm{~s}$ for venous phase scanning. CT images were exported in DICOM format.

\section{Pancreatic Texture Analysis}

Method of selecting ROls as described in Fig. 1B. Venous phase CT images had three ROls: the upper (upper layer) portion of the tumor, the maximum cross-sectional area of the tumor (middle layer), and the lower portion of the tumor (lower layer). All were selected and transferred to two personal computers for texture analysis. ${ }^{27}$ Subsequent image analysis utilized Photoshop (SC.6 Version, Adobe Corporation, USA) to segment the images. The pancreatic circumferential resection margin around the portal vein in the venous phase CT image was identified. Two physicians with 10 years of experience in abdominal CT diagnosis completed the delineation of all ROls, blinded to the histopathological status of the resection margin. To prevent measurement bias, the first physician sketched ROIs and performed feature extraction once, and then repeated the ROls sketching and feature extraction 2 weeks later, whilst another physician outlined ROls and feature extraction once to confirm concordance with the other physician.

\section{Pancreatic Texture Features}

Then we used the MATLAB software (2016b Version, MathWorks Corporation, USA) to extract the texture features by solving discrete Laplacian equations with Dirichlet boundary conditions, fit ROls to rectangular regions in Fig. 2. The extracted radiomics texture features including Histogram, Gray level cooccurrence matrix, Run-length matrix, Gabor transform, Wavelet transform are summarized in Table 1. 
Table 1

Extracted Texture Features

\begin{tabular}{|c|c|}
\hline Method & Feature name \\
\hline Histogram & $\begin{array}{l}\text { Mean; standard deviation; smoothness; cubic moment; uniformity; entropy; fourth } \\
\text { moment; }\end{array}$ \\
\hline $\begin{array}{l}\text { Gray level } \\
\text { co- } \\
\text { occurrence } \\
\text { matrix }\end{array}$ & Contrast; correlation; energy; uniformity; entropy; \\
\hline $\begin{array}{l}\text { Run-length } \\
\text { matrix }\end{array}$ & $\begin{array}{l}\text { Short run emphasis; long run emphasis; gray-level non-uniformity; run length non- } \\
\text { uniformity; run percentage; low gray-level run emphasis; high gray-level run emphasis; } \\
\text { short run low gray-level emphasis; short run high gray-level emphasis; long run low } \\
\text { gray-level emphasis; long run high gray-level emphasis; }\end{array}$ \\
\hline $\begin{array}{l}\text { Gabor } \\
\text { transform }\end{array}$ & Mean; variance; energy; \\
\hline $\begin{array}{l}\text { Wavelet } \\
\text { transform }\end{array}$ & Mean; variance; energy; \\
\hline
\end{tabular}

\section{Follow-up}

Outpatient and telephone follow-up were conducted to accurately record the postoperative survival of patients included in the study until December 2018. For some patients who were uncontactable, the national death registration database was checked.

\section{Statistical Analysis}

Based on the histopathological tumor resection margin status and CT images of postoperative disease examination results, we used the Mann-Whitney Utest to compare the texture characteristics of R0 resection margin and $\mathrm{R} 1$ resection margin. Receiver operating characteristic (ROC) curves and KaplanMeier analysis were correlated with the CT texture characteristics, > $1 \mathrm{~mm}$ and $\leq 1 \mathrm{~mm}$ resection margin and postoperative survival rate of the tumor to determine the influence of these factors on overall survival. Multivariate Cox proportional hazard regression analysis was performed to assess whether any of the important texture parameters were independent predictors of R0, R1 resection margin or overall survival. Spearman rank correlation method was used to evaluate the correlation amongst texture features.

\section{Statement}

All authors had access to the study data and reviewed and approved the final manuscript.

\section{Results}

\section{Patients}


Of the 215 patients with pancreatic head carcinoma who received pancreaticoduodenectomy screened, a total of 86 eligible patients were finally included and analyzed for radiomics (Fig. 3). There were 53 males and 33 females with mean age of $59.9 \pm 9.2$ years and $60.6 \pm 8.8$ years, respectively. According to postoperative pathological examination results, there were 52 patients in the $>1 \mathrm{~mm}$ resection margin group and 34 patients in the $\leq 1 \mathrm{~mm}$ resection margin group (carcinoma was found within $1 \mathrm{~mm}$ of the resection margin). The baseline parameters of patients in these two groups are show in Table 2. There was no statistical significance between the two groups $(P>0.05)$. 
Table 2

Baseline Characteristics of Included Patients

\begin{tabular}{|c|c|c|c|c|c|}
\hline & & $\begin{array}{l}\text { All patients } \\
(86)\end{array}$ & $\begin{array}{l}\text { Resection } \\
\text { margin } \\
>1 \mathrm{~mm}(52)\end{array}$ & $\begin{array}{l}\text { Resection } \\
\text { margin } \\
\leq 1 \mathrm{~mm}(34)\end{array}$ & $\stackrel{P}{\text { Value }}$ \\
\hline Age, years & $\begin{array}{l}\text { Mean } \pm \text { Standard } \\
\text { Deviation }\end{array}$ & $59.9 \pm 10.0$ & $60.8 \pm 8.8$ & $58.6 \pm 9.8$ & 0.304 \\
\hline Sex & & & & & 0.658 \\
\hline Male & $\mathrm{n}(\%)$ & $53(62)$ & $31(60)$ & $22(65)$ & \\
\hline Female & $\mathrm{n}(\%)$ & $33(38)$ & $21(40)$ & $12(35)$ & \\
\hline $\begin{array}{l}\text { Body mass index, } \\
\mathrm{kg} / \mathrm{m}^{2}\end{array}$ & $\begin{array}{l}\text { Mean } \pm \text { Standard } \\
\text { Deviation }\end{array}$ & $21.7 \pm 3.6$ & $\begin{array}{l}21.3(18.7 \\
23.2)\end{array}$ & $\begin{array}{l}21.6(19.9 \\
23.8)\end{array}$ & 0.365 \\
\hline $\begin{array}{l}\text { Diameter of } \\
\text { tumor, } \\
\mathrm{cm}\end{array}$ & Median (P25,P75) & $3.0(2.5,3.8)$ & $3.0(2.4,3.8)$ & $3.0(2.5,3.7)$ & 0.989 \\
\hline CA19-9, U/ml & Median (P25,P75) & $\begin{array}{l}102.6 \\
(33.6,415.1)\end{array}$ & $\begin{array}{l}266.4(39.8 \\
827.7)\end{array}$ & $\begin{array}{l}120.2(34.6 \\
791.7)\end{array}$ & 0.454 \\
\hline Hemoglobin, g/L & $\begin{array}{l}\text { Mean } \pm \text { Standard } \\
\text { Deviation }\end{array}$ & $118.4 \pm 23.1$ & $116.9 \pm 27.5$ & $120.4 \pm 13.7$ & 0.428 \\
\hline $\begin{array}{l}\text { Total bilirubin, } \\
\mu \mathrm{mol} / \mathrm{L}\end{array}$ & Median (P25,P75) & $\begin{array}{l}110.5 \\
(12.58,202.9)\end{array}$ & $\begin{array}{l}87.7(12.1, \\
191.1)\end{array}$ & $\begin{array}{l}150.4(16.5 \\
241.5)\end{array}$ & 0.351 \\
\hline $\begin{array}{l}\text { Pathological } \\
\text { grade }\end{array}$ & & & & & 0.594 \\
\hline Poor & $\mathrm{n}(\%)$ & $10(12)$ & $7(13)$ & $3(9)$ & \\
\hline Poor-Moderate & $\mathrm{n}(\%)$ & $38(44)$ & $20(38)$ & $18(53)$ & \\
\hline Moderate & $\mathrm{n}(\%)$ & $32(37)$ & $21(41)$ & $11(32)$ & \\
\hline Moderate-Well & $\mathrm{n}(\%)$ & $5(6)$ & $3(6)$ & $2(6)$ & \\
\hline Well & $\mathrm{n}(\%)$ & $1(1)$ & $1(2)$ & $0(0)$ & \\
\hline $\mathrm{T}$ & & & & & 0.586 \\
\hline 1 & $\mathrm{n}(\%)$ & $15(17)$ & $9(17)$ & $6(18)$ & \\
\hline 2 & $\mathrm{n}(\%)$ & $62(72)$ & $39(75)$ & $23(68)$ & \\
\hline 3 & $\mathrm{n}(\%)$ & $9(11)$ & $4(8)$ & $5(15)$ & \\
\hline$N$ & & & & & 0.713 \\
\hline$x$ & $\mathrm{n}(\%)$ & $10(12)$ & $4(8)$ & $6(18)$ & \\
\hline
\end{tabular}




\begin{tabular}{|llllll|}
\hline & & $\begin{array}{l}\text { All patients } \\
(\mathbf{8 6})\end{array}$ & $\begin{array}{l}\text { Resection } \\
\text { margin } \\
>1 \mathrm{~mm}(52)\end{array}$ & $\begin{array}{l}\text { Resection } \\
\text { margin } \\
\leq 1 \mathrm{~mm}(34)\end{array}$ & $\begin{array}{l}P \\
\text { Value }\end{array}$ \\
\hline 0 & $\mathrm{n}(\%)$ & $39(45)$ & $28(54)$ & $11(32)$ \\
\hline 1 & $\mathrm{n}(\%)$ & $26(30)$ & $14(26)$ & $12(35)$ & \\
\hline Stage & $\mathrm{n}(\%)$ & $11(13)$ & $6(12)$ & $5(15)$ & \\
\hline IA & & & & $3(9)$ & \\
\hline IB & $\mathrm{n}(\%)$ & $12(14)$ & $9(17)$ & $7(21)$ & \\
\hline IIA & $\mathrm{n}(\%)$ & $26(30)$ & $19(37)$ & $1(3)$ & \\
\hline IIB & $\mathrm{n}(\%)$ & $2(2)$ & $1(2)$ & $12(35)$ & \\
\hline III & $\mathrm{n}(\%)$ & $25(29)$ & $13(25)$ & $11(32)$ & \\
\hline $\begin{array}{l}\text { Adjuvant } \\
\text { chemotherapy }\end{array}$ & $\mathrm{n}(\%)$ & $21(25)$ & $10(19)$ & $8(24)$ & \\
\hline
\end{tabular}

No patients got neoadjucant therapy. Although, adjuvant therapy was strongly recommended to all patients after surgery, only 6 patients were treated adjuvant therapy based on Gemcitabine, 19 patients were treated with Tegafur Gimeracil Oteracil Potassium Capsule, 2 patients were treated with Tegafur Gimeracil Oteracil Potassium Capsule combined Gemcitabine. 1 patient were treated with FOLFIRINOX, 1 with Doxifluridine. 57 patients did not get any adjuvant therapy.

The follow-up period was 6-38 months, and the mean survival time was $19.3 \pm 1.1$ months. The overall three-year survival rate was $40.7 \%$ for all patients, $51.9 \%$ for $>1 \mathrm{~mm}$ resection margin patients, and $23.5 \%$ for $\leq 1 \mathrm{~mm}$ resection margin patients. Kaplan-Meier survival curve for patients with resection margin > $1 \mathrm{~mm}$ and $\leq 1 \mathrm{~mm}$ is shown in Fig. 4A. Of the 86 patients, 5 were lost to follow-up. Intend-to-treat analysis revealed that of the 52 cases with $>1 \mathrm{~mm}$ resection margin, the mean survival time was $21.4 \pm$ 1.4 months (median $21 \pm 2.1$ months); in the 34 cases with resection margin $\leq 1 \mathrm{~mm}$, the mean survival time was $16.5 \pm 1.5$ months (median $16 \pm 1.1$ months).

Results of feature selection identifying the resection margin are listed in Table 3. There was no statistical significance between the comparison of Histogram features between resection margin of $>1 \mathrm{~mm}$ and $\leq$ $1 \mathrm{~mm}(P>0.05)$. At $\mathrm{d}=1$ and 2 in the lower layer ROI, where " $\mathrm{d}$ " is the distance of the co-occurrence matrix and "diagonal" expresses the component direction of the Wavelet transform, the characteristic correlations of Gray level co-occurrence matrix was statistically significant $(P<0.05)$, with the remainder demonstrating no statistical significance $(P>0.05)$. The characteristic high Gray-level run emphasis of Run-length matrix within the middle layer ROI was statistically significant $(P<0.05)$, with the remainder demonstrating no statistical significance $(P>0.05)$. The characteristic average filter of Wavelet transform 
within the lower layer ROI was statistically significant $(P<0.05)$, with the remainder demonstrating no statistical significance $(P>0.05)$

Table 3

\begin{tabular}{|c|c|c|c|c|}
\hline \multicolumn{5}{|c|}{ Results of Feature Selection to Identify the Resection Margin } \\
\hline Location & Selected feature & AUC & $95 \% \mathrm{Cl}$ & $\mathrm{P}$ Value \\
\hline Upper & \multicolumn{4}{|l|}{ None } \\
\hline Middle & Run-length matrix, high gray-level run emphasis & 0.626 & $0.505-0.746$ & 0.050 \\
\hline \multirow[t]{3}{*}{ Lower } & $\begin{array}{l}\text { Gray level co-occurrence matrix, } d=1 \text {, } \\
\text { correlation; }\end{array}$ & 0.660 & $0.542-0.778$ & 0.012 \\
\hline & $\begin{array}{l}\text { Gray level co-occurrence matrix, } d=2 \text {, } \\
\text { correlation; }\end{array}$ & 0.635 & $0.515-0.754$ & 0.035 \\
\hline & Wavelet transform, average filter & 0.656 & $0.539-0.773$ & 0.015 \\
\hline All & Four features combination & 0.784 & $0.689-0.879$ & 0.000 \\
\hline \multicolumn{5}{|c|}{$\begin{array}{l}\text { Multivariate Cox Proportional Hazards Regression Analysis and Independent Predictors of Prognosis } \\
\text { with Resection Margin }\end{array}$} \\
\hline Location & Selected feature & AUC & $95 \% \mathrm{Cl}$ & $\mathrm{P}$ Value \\
\hline Upper & Wavelet transform, energy; & 0.998 & $0.995-1.000$ & 0.036 \\
\hline \multirow[t]{4}{*}{ Middle } & Histogram, measure of smoothness; & 0.000 & $0.000-0.077$ & 0.045 \\
\hline & Gabor transform, variance & 0.241 & $0.099-0.587$ & 0.002 \\
\hline & (1 direction); & & & \\
\hline & $\begin{array}{l}\text { Gabor transform, variance } \\
\text { ( } 2 \text { direction); }\end{array}$ & 51.297 & $\begin{array}{l}2.187- \\
1203.140\end{array}$ & 0.014 \\
\hline Lower & \multicolumn{4}{|l|}{ None } \\
\hline
\end{tabular}

ROC curve (Fig. 4B) showing that $>1 \mathrm{~mm}$ resection margins are differentiated from $\leq 1 \mathrm{~mm}$ resection margins based on four characteristic radiomic features. The area under the curve (AUC) was 0.784 $(95 \% \mathrm{Cl} 0.689-0.879)$ at sensitivity of $79.4 \%$ and specificity of $69.2 \%$.

Multivariate Cox proportional hazards regression analysis showed that the energy of Wavelet transform in the upper layer, the measure of smoothness of Histogram and the variance in 2 direction of Gabor transform in the middle layers are independent predictors of overall survival prognosis independent of resection margin (Table 3) 
Spearman correction analysis of characteristic radiomics features for resection margin and prognosis (Table 4). In the feature selection to identify the resection margin, the Gray level co-occurrence matrix of the lower layer, correlation of distance 1 and 2 were strongly correlated $(P<0.05)$. In the selected feature of independent predictors for prognosis, the four characteristic features were strongly correlated with each other $(P<0.05)$. 
Table 4

\section{Spearman Rank Correlation for Resection Margin Features}

\begin{tabular}{|c|c|c|c|c|}
\hline \multirow[t]{2}{*}{$\begin{array}{l}\text { Resection Margin } \\
\text { Features }\end{array}$} & \multirow{2}{*}{$\begin{array}{l}\text { Gray level co- } \\
\text { occurrence matrix, } \\
d=1 \\
\text { correlation }\end{array}$} & \multirow{2}{*}{$\begin{array}{l}\text { Gray level co- } \\
\text { occurrence matrix, } \\
d=2 \\
\text { correlation }\end{array}$} & \multirow{2}{*}{$\begin{array}{l}\text { Run-length } \\
\text { matrix } \\
\text { high gray-level } \\
\text { run emphasis }\end{array}$} & \multirow{2}{*}{$\begin{array}{l}\text { Wavelet } \\
\text { transform } \\
\text { average } \\
\text { filter }\end{array}$} \\
\hline & & & & \\
\hline $\begin{array}{l}\text { Gray level co- } \\
\text { occurrence matrix, } d \\
=1\end{array}$ & - & $0.964(0.000)$ & $-0.117(0.285)$ & $\begin{array}{l}0.067 \\
(0.540)\end{array}$ \\
\hline
\end{tabular}

correlation

Gray level co-

$0.964(0.000)$

$\begin{array}{ll}- & -0.092(0.400)\end{array}$

0.067

occurrence matrix, $\mathrm{d}$

$(0.540)$

$=2$

correlation

Run-length matrix

$-0.117(0.285)$

$-0.092(0.400)$

0.020

high gray-level run

$(0.857)$

emphasis correlation

Wavelet transform

$0.067(0.540)$

$0.067(0.540)$

$0.020(0.857)$

average filter

Spearman Rank Correlation for Prognosis Features

\begin{tabular}{|c|c|c|c|c|}
\hline Prognosis Features & $\begin{array}{l}\text { Histogram, } \\
\text { measure of } \\
\text { smoothness }\end{array}$ & $\begin{array}{l}\text { Gabor transform } \\
\text { variance } \\
\text { (1 direction) }\end{array}$ & $\begin{array}{l}\text { Gabor } \\
\text { transform, } \\
\text { variance } \\
\text { (2 direction) }\end{array}$ & $\begin{array}{l}\text { Wavelet } \\
\text { transfor } \\
\text { energy }\end{array}$ \\
\hline Histogram, & - & $0.434(0.000)$ & $0.532(0.000)$ & $\begin{array}{l}0.667 \\
(0.000)\end{array}$ \\
\hline
\end{tabular}

smoothness

Gabor transform

$0.434(0.000)$

$0.871(0.000)$

0.353

$(0.001)$

variance

(1 direction)

Gabor transform

$0.532(0.000)$

$0.871(0.000)$

0.446

variance

(0.000)

(2 direction)

Wavelet transform

$0.667(0.000)$

$0.353(0.001)$

$0.446(0.000)$

energy 


\section{Spearman Rank Correlation for Resection Margin Features}

\section{$P$ value in bracket}

\section{Discussion}

In this study, we provide a new means of preoperative assessment based on radiomics for preoperative prediction of resection margin status prior to pancreaticoduodenectomy for pancreatic head adenocarcinoma. We demonstrate that the radiomics labels displaying significant predictive power for the recognition of the resection margin of the pancreatic ahead adenocarcinoma ( $>1 \mathrm{~mm} v \mathrm{vs} \leq 1 \mathrm{~mm}$ ) were Gray level co-occurrence matrix, Run-length matrix and Wavelet transform. These findings are of significant potential importance for the treatment of pancreatic head adenocarcinoma in the context of previous studies that have identified no difference in survival rate between patients with resection margin of $\leq 1 \mathrm{~mm}$ and patients with an R1 resection and the patients might benefit from neoadjucant therapy, especially for the patients with lymph node positive. ${ }^{8}$ Based on the radiomic parameters established in our study, we can accurately identify the resection margin in the majority of patients before surgery, identifying a potential subgroup of patients that may benefit from neoadjuvant chemotherapy first, rather than directly receiving surgery.

This study confirmed that the resection margin status of resected pancreatic head adenocarcinoma was correlated with worse overall survival $(P<0.05)$. The overall three-year survival rate was $40.7 \%$ for all patients, $51.9 \%$ for $>1 \mathrm{~mm}$ resection margin patients, and $23.5 \%$ for $\leq 1 \mathrm{~mm}$ resection margin patients. The overall three-year survival rate in our study was not good as other report. ${ }^{8}$ We thought that might cause by inadequate adjuvant chemotherapy. Most patients involved in this study came from west China. Some patients refused adjuvant chemotherapy because they were afraid of adverse reactions of adjuvant chemotherapy. Some patients did not believe that they could get benefits from adjuvant chemotherapy. Thus, only $40 \%$ patients were treated with adjuvant chemotherapy. Even though most patients did not treat with adjuvant chemotherapy, resection margin status was also an independent prognostic factor for patients with pancreatic head adenocarcinoma. In the past, the clinical judgment criteria for resection margin status of pancreatic head adenocarcinoma specimens has been inconsistent. In most cases, the absence or presence of tumor cells on the resection margin surface has been used as the basis for R0 or $\mathrm{R} 1$ resection. ${ }^{10}$ As a result, the difference in local recurrence rate and prognosis between R0 and R1 resection patients has occasionally been reported as not statistically significant. ${ }^{28}$ Verbeke et al. proposed a new international standard based on the "1 $\mathrm{mm}$ principle" for R0 resection of resected specimens with circumferential resection margin greater than or equal to $1 \mathrm{~mm} .{ }^{29-31}$ The "1 mm principle" means that no tumor cells within a resection margin greater than $1 \mathrm{~mm}$ shall be classified as $\mathrm{R} 0$, with the remainder classified as R1. ${ }^{27,29,31}$ Strobel et al. also evaluated the data of 561 patients with pancreatic head adenocarcinoma who underwent pancreaticoduodenectomy by using the "1 mm principle", the results showed that $80 \%$ of the patients underwent R1 resection, while only $20 \%$ underwent $\mathrm{R} 0$ resection, and the difference in prognosis between the two groups was statistically significant. ${ }^{10,32}$ 
Although the "1 $\mathrm{mm}$ principle" pointed out the limitations of previous surgical treatment of pancreatic cancer, it remains very difficult to evaluate this significant prognostic factor before surgery, with no current imaging studies currently reported in the literature focused on the resection margin of pancreatic head adenocarcinoma.

Due to the biological characteristics of the tumor itself, there are differences between different texture features in enhanced CT images. However, many of the differences visible on CT images may reflect the radiologists' subjective impression of image quality, which can mask any underlying biologic heterogeneity. ${ }^{33}$ Using CT texture analysis, these image features can be detected and objectively quantified. ${ }^{34}$

The normal pancreatic tissue lacks an obvious capsule and has abundant adipose tissue. ${ }^{35}$ However, in pancreatic cancer, there is more stroma in the tissue, which is prone to causing fibrosis of surrounding tissues and associated poor blood supply. ${ }^{36}$ These key tumor biological characteristics can be detected by enhanced CT. ${ }^{37}$ In this study, we collected the texture characteristics data from 258 portal venous phase images of 86 patients with pancreatic head adenocarcinoma. The ROls we selected for the resection margin included normal pancreatic tissue and tumor tissue for radiomics methods analysis. We analyzed the images of the resection margin at three layers in patients with pancreatic head adenocarcinoma, rather than the entire tumor at a single layer as in other studies, ${ }^{38,39}$ as different resection margins could reflect different tumor biological characteristics present within the heterogenous primary tumor. This makes our study more detailed and representative compared to other studies. ${ }^{40,41}$ Our results showed correlations of Gray level co-occurrence matrix, high Gray-level run emphasis of Runlength matrix and average filter of Wavelet transform with resection margin status ( $>1 \mathrm{~mm}$ vs $\leq 1 \mathrm{~mm}$ ). Gray level co-occurrence matrix is a texture analysis form that provides statistical measurement of spatial relationship of pixels in images, ${ }^{11}$ whilst the run length matrix is the length of the continuum element with the same gray level in the preset direction. ${ }^{42}$ The grayscale run length is only a measurement of image pixel information. In actual practice, it is also necessary to calculate the generated grayscale run length matrix and obtain further image feature information based on the grayscale co-occurrence matrix. The relationship between run lengths produces textures and Wavelet transform is a means of transforming space (time) and frequency, to provide a more effective signal analysis, ${ }^{22}$ advantageous as it allows the image to be analyzed at multiple scales. ${ }^{22}$ In this study, the AUC of four characteristic features was 0.784 , sensitivity was $75 \%$ and specificity was $79 \%$, allowing accurate prediction of $\leq 1 \mathrm{~mm}$ resection margins. However, this study also has some limitations. All the extracted features in this study are two-dimensional features of CT images, and should ideally be the features of the entire three-dimensional CT image of the resection margin, so as to provide a more comprehensive assessment of the tumor and potential resection margin status.

\section{Conclusions}


Radiomic texture analysis of pre-operative enhanced CT images can be used for accurate preoperative assessment of resection margins in patients with pancreatic ahead adenocarcinoma providing clinicians alongside patients a more refined non-invasive means of perioperative prognostication to guide management.

\section{List Of Abbreviations}

CT = Computed Tomography

$\mathrm{ROI}=$ Regions Of Interest

AUC=Area Under the Curve

$\mathrm{RCT}=$ Randomized controlled trials

ROC=Receiver operating characteristic

\section{Declarations}

\section{Ethics approval and consent to participate}

The study protocol was approved by the Ethics Committee of West China Hospital of Sichuan University (2016:122). And the patients had consented to participate in this study, and the consent obtained was written.

\section{Consent for publication}

Not Applicable. And the patients had consented for publication.

\section{Availability of data and materials}

The datasets used and analysed during the current study available from the corresponding author on reasonable request.

\section{Competing interests}

The authors declare that they have no competing interests

\section{Funding}

1, The National Natural Science Foundation of China (81602133) was for writing the manuscript. 
2, The 1.3.5 project for disciplines of excellence, West China Hospital, Sichuan University (ZY2017302 13-5) was for the design of the study.

3, The Scientific and Technological Support Program of Sichuan Province (2016FZ0115) was for collection, analysis, and interpretation of data.

\section{Authors' contributions}

$\mathrm{JHL}$ and XBL contributed equally to this study.

NWK and XBL designed the research.

TYW and JHL reviewed existing publications, extracted the materials, and wrote the manuscript. JJQ collected the data.

WH edited the manuscript.

All authors read and approved the final manuscript.

\section{Acknowledgements}

The authors thanked the pancreas multi-disciplinary team members for their continuous support. And thanks Professor Zixing Huang for providing purely technical help.

\section{References}

1. Xiao AY, Tan ML, Wu LM, et al. Global incidence and mortality of pancreatic diseases: a systematic review, meta-analysis, and meta-regression of population-based cohort studies. Lancet Gastroenterol Hepatol 2016;1:45-55.

2. Zhou $M$, Wang $H$, Zeng $X$, et al. Mortality, morbidity, and risk factors in China and its provinces, 19902017: a systematic analysis for the Global Burden of Disease Study 2017. Lancet 2019.

3. Siegel RL, Miller KD, Jemal A. Cancer statistics, 2019. CA Cancer J Clin 2019;69:7-34.

4. Greenlee RT, Hill-Harmon MB, Murray T, et al. Cancer statistics, 2001. CA Cancer J Clin 2001;51:15-36.

5. Ryan DP, Hong TS, Bardeesy N. Pancreatic adenocarcinoma. N Engl J Med 2014;371:1039-49.

6. Bockhorn M, Uzunoglu FG, Adham M, et al. Borderline resectable pancreatic cancer: a consensus statement by the International Study Group of Pancreatic Surgery (ISGPS). Surgery 2014;155:977-88.

7. Tempero MA, Malafa MP, Behrman SW, et al. Pancreatic adenocarcinoma, version 2.2014: featured updates to the NCCN guidelines. J Natl Compr Canc Netw 2014;12:1083-93.

8. Delpero JR, Jeune F, Bachellier P, et al. Prognostic Value of Resection Margin Involvement After Pancreaticoduodenectomy for Ductal Adenocarcinoma: Updates From a French Prospective Multicenter Study. Ann Surg 2017;266:787-796. 
9. Delpero JR, Bachellier P, Regenet N, et al. Pancreaticoduodenectomy for pancreatic ductal adenocarcinoma: a French multicentre prospective evaluation of resection margins in 150 evaluable specimens. HPB 2014, 16, 20-33

10. Strobel O, Hank T, Hinz U, et al. Pancreatic Cancer Surgery: The New R-status Counts. Ann Surg 2017;265:565-573.

11. Yun G, Kim YH, Lee YJ, et al. Tumor heterogeneity of pancreas head cancer assessed by CT texture analysis: association with survival outcomes after curative resection. Sci Rep 2018;8:7226.

12. Wang $S$, Meng $M$, Zhang $X$, et al. Texture analysis of diffusion weighted imaging for the evaluation of glioma heterogeneity based on different regions of interest. Oncol Lett 2018;15:7297-7304.

13. Moon SH, Kim J, Joung JG, et al. Correlations between metabolic texture features, genetic heterogeneity, and mutation burden in patients with lung cancer. Eur J Nucl Med Mol Imaging 2019;46:446-454.

14. Ren J, Yuan Y, Shi Y, et al. Tumor heterogeneity in oral and oropharyngeal squamous cell carcinoma assessed by texture analysis of CT and conventional MRI: a potential marker of overall survival. Acta Radiol 2019:284185119825487.

15. Lambin P, Rios-Velazquez E, Leijenaar R, et al. Radiomics: extracting more information from medical images using advanced feature analysis. Eur J Cancer 2012;48:441-6.

16. Aerts HJ, Velazquez ER, Leijenaar RT, et al. Decoding tumour phenotype by noninvasive imaging using a quantitative radiomics approach. Nat Commun 2014;5:4006.

17. Hwang IP, Park CM, Park SJ, et al. Persistent Pure Ground-Glass Nodules Larger Than 5 mm: Differentiation of Invasive Pulmonary Adenocarcinomas From Preinvasive Lesions or Minimally Invasive Adenocarcinomas Using Texture Analysis. Invest Radiol 2015;50:798-804.

18. Skorton DJ, Collins SM, Nichols J, et al. Quantitative texture analysis in two-dimensional echocardiography: application to the diagnosis of experimental myocardial contusion. Circulation 1983;68:217-23.

19. Rizzo S, Botta F, Raimondi S, et al. Radiomics: the facts and the challenges of image analysis. Eur Radiol Exp 2018;2:36.

20. Lee G, Bak SH, Lee HY. CT Radiomics in Thoracic Oncology: Technique and Clinical Applications. Nucl Med Mol Imaging 2018;52:91-98.

21. Bi WL, Hosny A, Schabath MB, et al. Artificial intelligence in cancer imaging: Clinical challenges and applications. CA Cancer J Clin 2019;69:127-157.

22. Zhang MM, Yang H, Jin ZD, et al. Differential diagnosis of pancreatic cancer from normal tissue with digital imaging processing and pattern recognition based on a support vector machine of EUS images. Gastrointest Endosc 2010;72:978-85.

23. Canellas R, Burk KS, Parakh A, et al. Prediction of Pancreatic Neuroendocrine Tumor Grade Based on CT Features and Texture Analysis. AJR Am J Roentgenol 2018;210:341-346. 
24. Li J, Lu J, Liang P, et al. Differentiation of atypical pancreatic neuroendocrine tumors from pancreatic ductal adenocarcinomas: Using whole-tumor CT texture analysis as quantitative biomarkers. Cancer Med 2018;7:4924-4931.

25. Guo C, Zhuge X, Wang Z, et al. Textural analysis on contrast-enhanced CT in pancreatic neuroendocrine neoplasms: association with WHO grade. Abdom Radiol (NY) 2019;44:576-585.

26. van der Pol CB, Lee $S$, Tsai $S$, et al. Differentiation of pancreatic neuroendocrine tumors from pancreas renal cell carcinoma metastases on CT using qualitative and quantitative features. Abdom Radiol (NY) 2019;44:992-999.

27. Verbeke CS, Menon KV. Redefining resection margin status in pancreatic cancer. HPB (Oxford) 2009;11:282-9.

28. Kim KS, Kwon J, Kim K, et al. Impact of Resection Margin Distance on Survival of Pancreatic Cancer: A Systematic Review and Meta-Analysis. Cancer Res Treat 2017;49:824-833.

29. Verbeke CS, Leitch D, Menon KV, et al. Redefining the R1 resection in pancreatic cancer. Br J Surg 2006;93:1232-7.

30. Verbeke CS. Resection margins and R1 rates in pancreatic cancer-are we there yet? Histopathology 2008;52:787-96.

31. Verbeke CS. Resection margins in pancreatic cancer. Pathologe 2013;34 Suppl 2:241-7.

32. Strobel O, Hartwig W, Bergmann F, et al. Anaplastic pancreatic cancer: Presentation, surgical management, and outcome. Surgery 2011;149:200-8.

33. Fabbri C, Bartolomei M, Mattone V, et al. (90)Y-PET/CT Imaging Quantification for Dosimetry in Peptide Receptor Radionuclide Therapy: Analysis and Corrections of the Impairing Factors. Cancer Biother Radiopharm 2015;30:200-10.

34. Ganeshan B, Goh V, Mandeville HC, et al. Non-small cell lung cancer: histopathologic correlates for texture parameters at CT. Radiology 2013;266:326-36.

35. Syed AB, Mahal RS, Schumm LP, et al. Pancreas size and volume on computed tomography in normal adults. Pancreas 2012;41:589-95.

36. Zhang L, Sanagapalli S, Stoita A. Challenges in diagnosis of pancreatic cancer. World J Gastroenterol 2018;24:2047-2060.

37. Brook OR, Brook A, Vollmer CM, et al. Structured reporting of multiphasic CT for pancreatic cancer: potential effect on staging and surgical planning. Radiology 2015;274:464-72.

38. Sandrasegaran K, Lin Y, Asare-Sawiri M, et al. CT texture analysis of pancreatic cancer. Eur Radiol 2019;29:1067-1073.

39. Guo C, Zhuge $X$, Wang Q, et al. The differentiation of pancreatic neuroendocrine carcinoma from pancreatic ductal adenocarcinoma: the values of CT imaging features and texture analysis. Cancer Imaging 2018;18:37.

40. Gilbert JW, Wolpin B, Clancy T, et al. Borderline resectable pancreatic cancer: conceptual evolution and current approach to image-based classification. Ann Oncol 2017;28:2067-2076. 
41. Attiyeh MA, Chakraborty J, Doussot A, et al. Survival Prediction in Pancreatic Ductal Adenocarcinoma by Quantitative Computed Tomography Image Analysis. Ann Surg Oncol 2018;25:1034-1042.

42. Molina D, Perez-Beteta J, Luque B, et al. Tumour heterogeneity in glioblastoma assessed by MRI texture analysis: a potential marker of survival. Br J Radiol 2016;89:20160242.

\section{Figures}
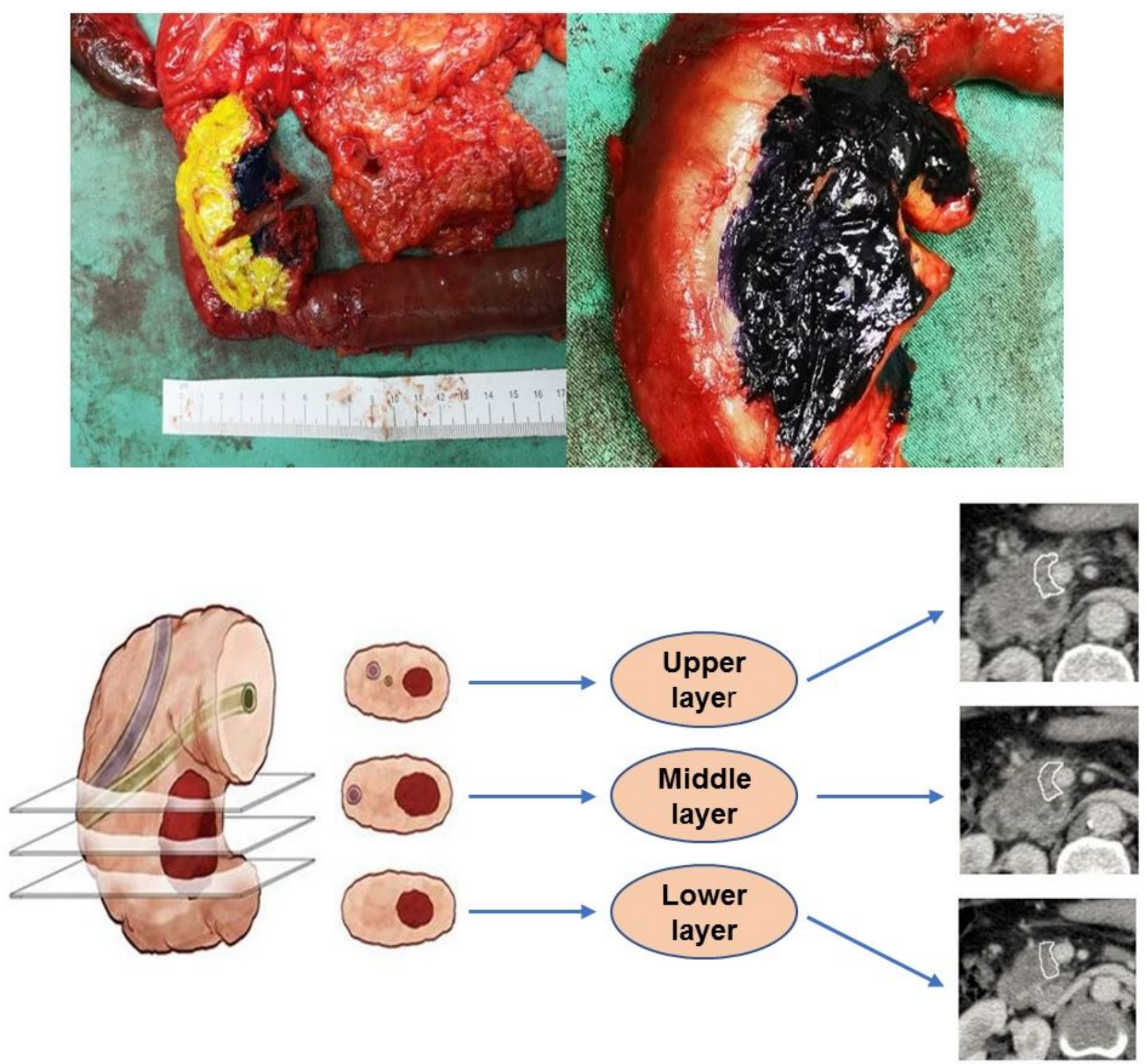

Figure 1 
Pathological staining of resection margin and selection of surgical region of interest from CT imaging. (A) Pathological resection margin staining for pancreatic head carcinoma. (B) Surgical region of interest selection from abdominal CT imaging. CT, computed tomography.

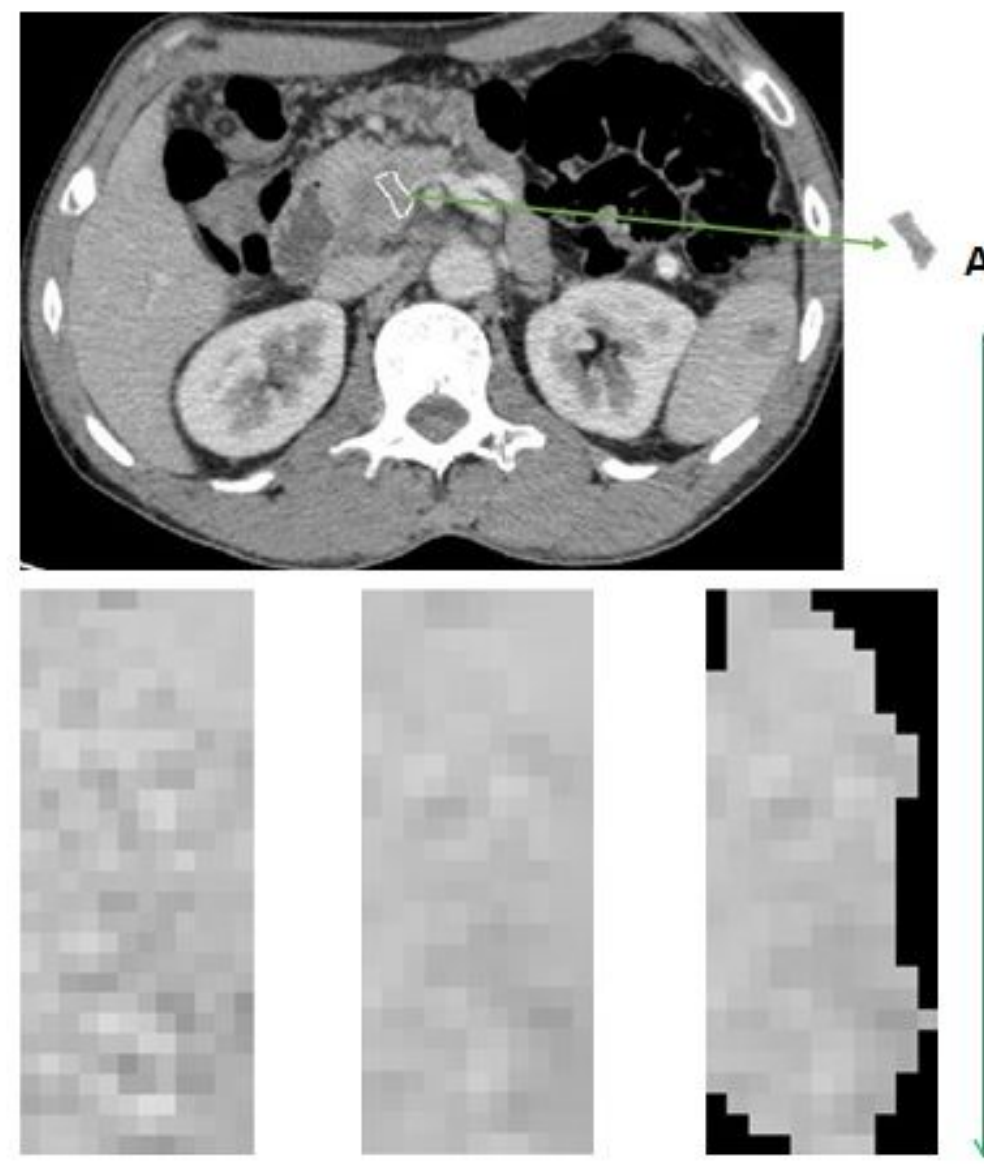

C

B

Figure 2

Extracted the texture features by solving discrete Laplacian equations with Dirichlet boundary conditions, fit ROls to rectangular regions. 
Patients who underwent pancreaticoduodenectomy screened:

$$
n=215
$$

Postoperative pathological diagnosis

of pancreatic head carcinoma:

$$
n=137
$$

Patients who were with complete preoperative CT images:

$$
n=109
$$

\section{Excluded \\ Preoperative $C T$ scan was not performed in our hospital or only having MRI scan: $n=28$}

\section{Figure 3}

Patient selection flow chart.

$$
\begin{gathered}
\text { Excluded } \\
\text { Poor quality of imaging or } \\
\text { non-standardized CT scan: } \\
n=23
\end{gathered}
$$

Patients included in the study.

$$
n=86
$$



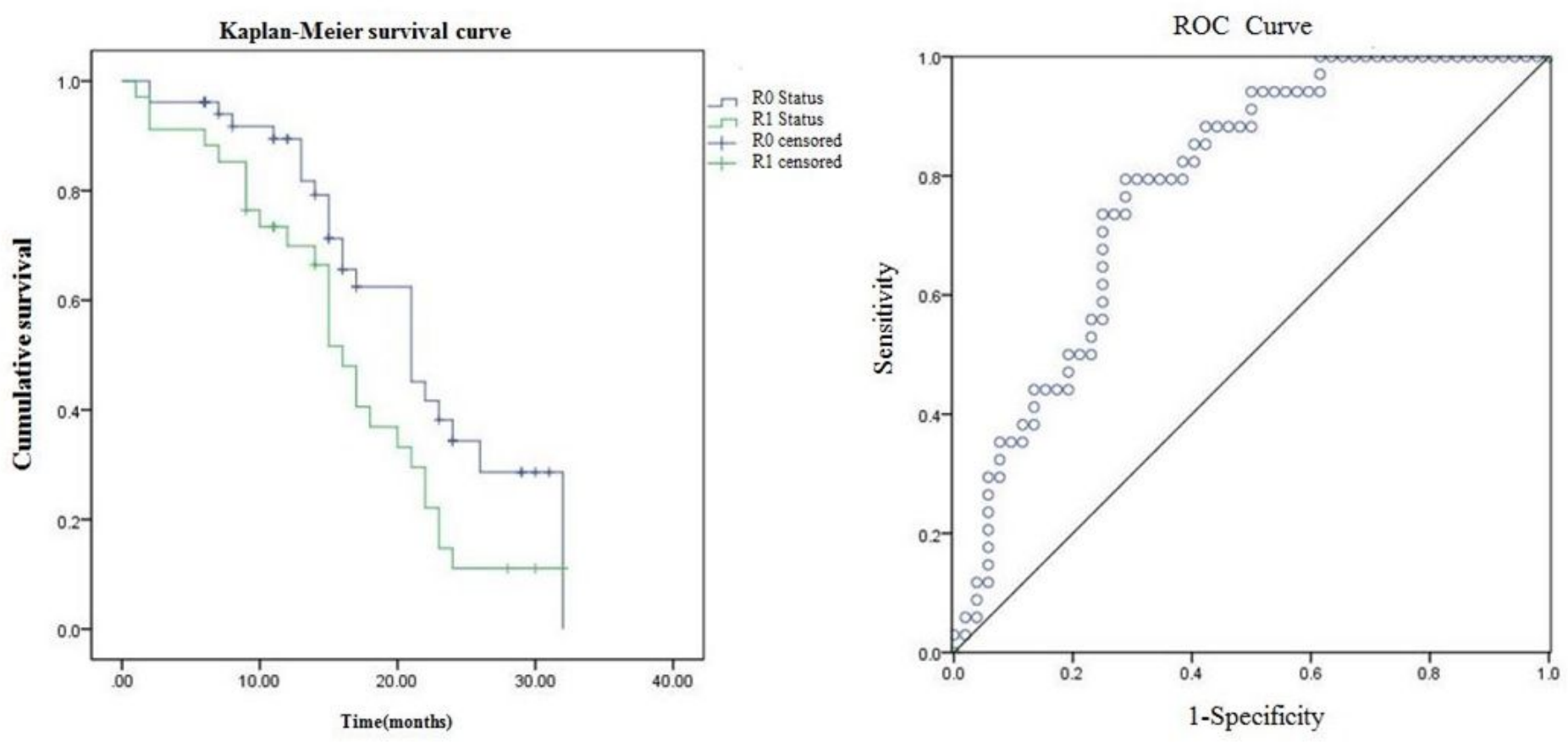

Figure 4

Kaplan-Meier survival curve for pancreatic head carcinoma resection margin of $>1 \mathrm{~mm}$ vs $\leq 1 \mathrm{~mm}$ and ROC curve for four characteristic radiomics features for resection margin. (A) Kaplan-Meier survival curve. (B) ROC curve. ROC, receiver operating characteristic. 